\title{
A critical appraisal of the SED fitting method to estimate photometric redshifts
}

\author{
M. Massarotti ${ }^{1,2}$, A. Iovino ${ }^{1}$, and A. Buzzoni ${ }^{1,3}$ \\ 1 Osservatorio Astronomico di Brera, Via Brera 28, 20121 Milano, Italy \\ 2 Università di Napoli, Dipartimento di Scienze Fisiche, Mostra d'Oltremare, 80125 Napoli, Italy \\ 3 Telescopio Nazionale Galileo, Roque de los Muchachos Astronomical Obs., PO Box 565, \\ 38700 Santa Cruz de La Palma (TF), Spain
}

Received 20 September 2000 / Accepted 12 December 2000

\begin{abstract}
We discuss the stability of the photometric redshift estimate obtained with the SED fitting method with respect to the choice of the galaxy templates. Within the observational uncertainty and photometric errors, we find satisfactory agreement among different sets of theoretical and empirical templates using the Hubble Deep Field North as a target galaxy sample. Our results suggest that, especially at high redshift, the description of the physical processes of photon absorption in the interstellar and intergalactic medium plays a dominant role in the redshift estimate. The specific choice of the template set, as long as this includes both normal and starburst galaxies, is in comparison a minor issue.
\end{abstract}

Key words. galaxies: distances and redshifts - galaxies: evolution - intergalactic medium - dust, extinction methods: data analysis - techniques: photometric

\section{Introduction}

Physical properties of high-redshift galaxies have been extensively explored in recent years thanks to a number of deep spectroscopic surveys carried out with the largest ground optical telescopes (e.g. Lilly et al. 1995; Cowie et al. 1996; Ellis et al. 1996). These surveys have enabled to trace well galaxy evolution up to $z \sim 1.5$.

Beyond this redshift limit, however, ground-based spectroscopy is forcedly confined to the brightest objects, a few magnitudes above the limit achieved by deep photometry. For the time being, spectra of galaxies fainter than $I \sim 24$ ab mag are still difficult to obtain, and one must therefore rely on multicolor observations coupled to photometric redshift techniques to estimate redshifts for the majority of galaxies in this magnitude range.

Photometric redshift techniques have already been applied by many authors to the ultra-deep images in the Hubble Deep Field North (HDFN, Williams et al. 1996), addressing in fine detail the study of the galaxy luminosity function, the star formation rate (SFR), the UV cosmological density, and the clustering properties (Madau et al. 1996; Connolly et al. 1997; Sawicki et al. 1997;

Send offprint requests to: M. Massarotti,

e-mail: massarot@brera.mi.astro.it;

iovino@brera.mi.astro.it; buzzoni@tng.iac.es
Franceschini et al. 1998; Pascarelle et al. 1998; Connolly et al. 1998; Miralles \& Pelló 1998; Arnouts et al. 1999).

Two main approaches have been used in the literature to measure photometric redshifts: the so-called trainingset method (e.g. Connolly et al. 1997; Wang et al. 1999), and the spectral energy distribution (SED) fitting method (e.g. Sawicki et al. 1997; Giallongo et al. 1998; Benítez 2000).

In the training-set method the relation between colors (sometimes also magnitudes) and redshift is calibrated using a spectroscopic galaxy sample. The accuracy of this method depends on the number and completeness of the reference templates, and obviously increases when acquiring new spectroscopic data. It becomes less reliable at high redshifts because of incompleteness and increasing redshift uncertainty of the most distant reference galaxies (see Benítez 2000; Fernández-Soto et al. 2000 for a full discussion of the method drawbacks).

In the SED fitting method, on the other hand, photometric redshift is obtained by comparing observed galaxy fluxes, $f_{i}^{\text {obs }}$ at the $i$ th photometric band, with a library of reference fluxes, $f_{i}^{\text {templ }}(z, T)$, depending $z$ on redshift, and $T$ a suitable set of parameters, that account for galaxy morphological type, age, metallicity, dust reddening etc. In this way, for each galaxy one can perform a $\chi^{2}$ confidence test obtaining the values of $z$ and $T$ that minimize flux residuals between observations and reference 
templates. In addition to the best- $z$ estimate, the method identifies an "optimum template", supplying information on galaxy age and morphology, although special caution must obviously be taken in using this piece of information, because of possible numerical degeneracies in the parameter space.

In this paper we will discuss in some detail the performance of the SED fitting method as a function of the template set chosen to reproduce galaxy colors. The primary aim is to assess the effects of different template properties on the numerical stability and physical consistency of the method. In our opinion, this "sanity check" must be preliminary to any direct comparison of photometric and spectroscopic redshift determinations, as different template sets can give statistically similar results when applied to the same spectroscopic subsample, but produce a different redshift distribution on the entire photometric catalog (Yee 1998).

The plan of the paper is as follows. We will first recall in Sect. 2 some key steps in the method. The theoretical and empirical template libraries are introduced in Sect. 3, while Sect. 4 presents results obtained by comparing the different galaxy template sets. The role of physical processes in the interstellar and intergalactic medium is discussed in Sect. 5, where we show their relevant impact on redshift estimate. Section 6 finally summarizes our conclusions.

\section{The SED fitting algorithm}

Before proceeding with our discussion, it might be useful to briefly remind how the SED fitting algorithm works.

As a first step one has to convert galaxy observed magnitudes for each $i$ th photometric band into incoming apparent flux, $f_{i}^{\text {obs }}(\lambda)$. This is equivalent to reconstruct the SED of target galaxies at very low spectral resolution by sampling their luminosity at the effective wavelength of the photometric bands available.

For each object, the sampled flux has then to be compared with the reference spectral libraries of template galaxies, $f_{i}^{\text {templ }}(z, T)$, computing a $\chi^{2}$ merit function of the fitting residuals such as

$\chi^{2}=\sum_{i=1}^{N} \frac{\left[f_{i}^{\text {obs }}-s f_{i}^{\text {temp }}(z, T)\right]^{2}}{\sigma_{i}^{2}}$.

In the equation, $N$ is the number of photometric bands, and $\sigma_{i}$ is the observational uncertainty in the $i$ th band. The scale factor $s$ is chosen in such a way as to minimize $\chi^{2}$ for each template:

$s=\frac{\sum_{i=1}^{N} f_{i}^{\mathrm{obs}} f_{i}^{\mathrm{temp}}(z, T) / \sigma_{i}^{2}}{\sum_{i=1}^{N} f_{i}^{\mathrm{temp}}(z, T)^{2} / \sigma_{i}^{2}}$

We will search the galaxy redshift in the range $z \in$ $[0.0,5.0]$ with a step of 0.02 .

As in the current literature, a further constraint has been added to the fit imposing that the age of the best template cannot be older than the Hubble time at the selected redshift (in our calculations we assumed a $H_{0}=$ $50 \mathrm{~km} \mathrm{~s}^{-1} \mathrm{Mpc}^{-1}, q_{0}=0.0$ cosmology with $\Lambda=0$ ). It can be verified, however, that redshift estimates do not depend critically on the details of the cosmological model, and this constraint could be dropped with no serious impact on the final output.

\section{Theoretical vs. empirical galaxy templates}

A key issue in the SED fitting method is the selection of the reference flux library. In particular, one has to decide whether it is more appropriate to use empirical or synthetic galaxy templates.

The major advantage of the first choice is that the observed SEDs for a suitable set of local galaxies, spanning the whole range of Hubble morphological types, gives by definition a physically consistent picture of the real galaxies, at least the nearby ones. On the other hand, there is no obvious argument supporting a straightforward extension of the current galaxy properties to high-redshift objects. At earlier cosmological epochs, evolution could play a substantial role in changing both morphological and spectrophotometric properties of distant galaxies (see Buzzoni 1998 for a critical discussion on the subject).

In this respect, synthesis models should be preferred, as they do try to take into account evolution. Of course, some differences exist among current theoretical codes, especially in the SED predictions for the UV range (Charlot et al. 1996), and these differences should be carefully examined when trying to match high-redshift observations to derive $z$ from multicolor photometry. Optical (or even infrared) observations of high-redshift objects sample UV restframe emission.

We should also recall that at high $z$ the restframe wavelength range explored by a given photometric system becomes narrower. For example, at $z \sim 1$ the whole HST WFPC2 photometric system (i.e. $U_{300}, B_{450}, V_{606}$ plus the $I_{814}$ band) is just probing the wavelength range shortward of the galaxy $4000 \AA$ Balmer break, while for $z \sim 2$ the spectral window shrinks even more to a scarce $\pm 800 \AA$ interval around $1800 \AA$ in the galaxy restframe (cf. Fig. 1). Note in addition that, at least for early-type galaxies, SED drops to nominal values shortward of the Balmer discontinuity, so that $\kappa$ corrections become increasingly positive with $z$. As we can see in Fig. 1, the effect can reach up to two orders of magnitude for elliptical galaxies, and this can result in a strong bias against their detection at high redshifts.

Another problem to be addressed when comparing photometric observations of high-redshift galaxies with reference templates is the role of intervening interstellar (ISM) and intergalactic medium (IGM), which strongly modulates the observed galaxy SED. Interstellar dust attenuates the galaxy luminosity by an amount that greatly depends on the specific galaxy environment (Gordon et al. 1997; Bruzual et al. 1988; Kuchinski et al. 1998). The IGM, on the other hand, drastically absorbs galaxy 


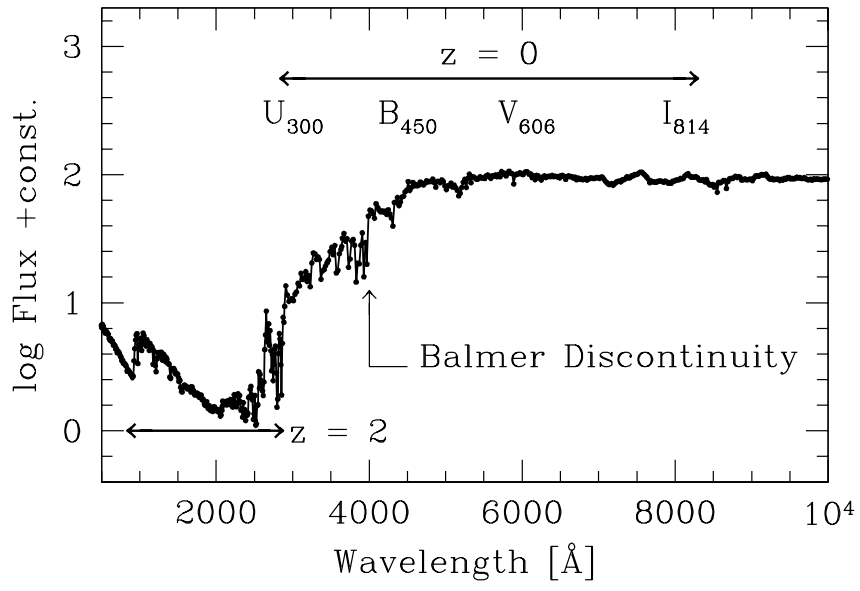

Fig. 1. Restframe portion of galaxy SED explored by the WFPC2 photometric system at $z=0$ and $z=2$. As an illustrative example a template model for local elliptical galaxies is shown

emission shortward of the Ly- $\alpha$ limit (the well known Ly$\alpha$ forest effect, e.g. Madau 1995). Unfortunately, present knowledge of these physical processes does not yet permit any firm and univocal correction of the data, leaving room for a variety of operational approaches (Sawicki et al. 1997; Giallongo et al. 1998; Miralles \& Pelló 1998).

In our study we will include three sets of theoretical galaxy models, namely those provided by the code of Bruzual \& Charlot (1993, hereafter BC), Buzzoni (1989, 1995, 2000; BUZ) and Fioc and Rocca-Volmerange (1997; FRV), as well as the empirical template set of Coleman et al. (1980; CWW) extensively used by previous authors (Sawicki et al. 1997; Fernández-Soto et al. 1999, hereafter FSLY; Benítez 2000; Thompson et al. 2000).

\subsection{Template libraries}

For each galaxy we are mainly interested in a measure of its redshift, but an age estimate and a morphological classification parameter are also a result of the SED fitting technique. In order to ensure an homogeneous sampling of the galaxy distinctive parameters, a selected grid of reference models for each of the three synthetic libraries has therefore been considered.

According to the leading physical processes in the galaxy stellar populations we can identify two main evolutionary regimes that induce relevant differences in galaxy luminosity:

i) "Early" galaxy evolution - In the first Gyr of life, most of the galaxy luminosity is provided by short-living stars of high mass $\left(M \geq 2 M_{\odot}\right.$, Buzzoni 2000). These are strong UV emitters and dominate galaxy luminosity at short wavelength. Given their short lifetime, these stars track actual star formation activity in the host galaxy through a measure of its integrated UV flux. Synthesis models all converge toward a unified starburst scenario for $t<1$ Gyr, despite any later morphological evolution that could then differentiate among spirals, ellipticals and irregulars (Buzzoni 1998; Leitherer et al. 1999).

ii) "Late" galaxy evolution - It comprises all the objects older than 1 Gyr, displaying morphological features accounted for by the standard Hubble classification. Disregarding any specific detail of star formation and morphology, the stellar bulk in old galaxies is dominated by stars with $M \leq 2 M_{\odot}$ experiencing a full redgiant-branch (RGB) phase tipping with a degenerate-core Helium flash (Renzini \& Buzzoni 1986).

For each of our synthesis codes, we first set up an "oldgalaxy" template library, tuning up reference models for present-day galaxies along the whole Hubble sequence, and consistently tracking back-in-time evolution up to $t=1$ Gyr. For ages less than 1 Gyr all our synthesis codes have been complemented by the starburst models at $t=50,100,500$, and 800 Myr from Leitherer et al. (1999), better suitable to describe high-mass stellar evolution.

Note that having used Leitherer et al. (1999) starburst models to describe young galaxies does not influence our results, as all the codes do actually converge, for early ages, to the redshift results provided by the Leitherer et al. (1999) models.

An important issue to optimize computing resources and prevent redundant oversampling in the parameter space is the age step in the grid of template models.

Based on the theory of simple stellar populations (cf. e.g. Buzzoni 1989) we know that luminosity evolution proceeds in this case like $L \propto t^{-\beta}$, with $\beta$ varying in the range between unity at UV wavelength and 0.7 in the infrared ( $K$ band). Even accounting for a smoother evolution, e.g. for a continuous SFR, a $\Delta t / t \sim 0.3$ age sampling is sufficient to confidently appreciate any $0.1 \mathrm{mag}$ change in apparent colors for high- $z$ spirals and ellipticals.

The adopted BC templates consist of models with four different SFR histories to match today's $E / S O, S a / S b$, $S c / S d$, and Im Hubble types. From the BUZ and FRV codes, we selected the original templates for $E, S b, S c$, Im morphological types. The BC models assume a Scalo IMF, while the FRV models assume a Rana \& Basu (1992) mass distribution, and the BUZ models adopt a standard Salpeter IMF. For all the models we sampled the different Hubble types at $t=1.0,1.4,2.0,4.0,8.0,10.0,12.0$ and 15.0 Gyr.

Table 1 gives a complete summary of the relevant distinctive properties of each theoretical library, reporting also the expected colors for present-day galaxies of different Hubble type.

The original CWW empirical template set consisted of four observed galaxy spectra, $E, S b c, S c d$ and $I m$ respectively. Sawicki et al. (1997) and Benítez (2000) had already noticed that adding starburst templates (sensibly bluer than any Im SED) to CWW greatly alleviates the systematic discrepancies found in redshift distribution of target galaxies beyond $z>1.5$. Similarly, the lack of starburst templates could have biased FSLY results by inducing an artificial peak in the galaxy distribution around $z \sim 1.7$. In this work, following the strategy adopted by 
Table 1. Galaxy templates from the reference synthesis codes and from Coleman et al. (1980)

\begin{tabular}{|c|c|c|c|c|c|c|c|}
\hline Template & $\begin{array}{c}\text { Age } \\
(z=0)\end{array}$ & $U-V$ & $B-V$ & $V-K$ & SFR & $\operatorname{IMF}^{(*)}$ & Remarks \\
\hline \multicolumn{8}{|c|}{ Bruzual \& Charlot (1993): } \\
\hline $\begin{array}{l}\text { E/S0 } \\
\text { Sab } \\
\text { Scd } \\
\text { Im }\end{array}$ & $\begin{array}{l}12 \mathrm{Gyr} \\
12 \mathrm{Gyr} \\
12 \mathrm{Gyr} \\
1.4 \mathrm{Gyr}\end{array}$ & $\begin{array}{l}1.58 \\
1.09 \\
0.78 \\
0.13\end{array}$ & $\begin{array}{l}1.00 \\
0.81 \\
0.66 \\
0.28\end{array}$ & $\begin{array}{l}3.22 \\
2.95 \\
2.74 \\
1.89\end{array}$ & $\begin{array}{c}\propto \exp (-t / \tau), \tau=1 \mathrm{Gyr} \\
\propto \exp (-t / \tau), \tau=4 \mathrm{Gyr} \\
\propto \exp (-t / \tau), \tau=15 \mathrm{Gyr} \\
\text { const. }\end{array}$ & $\begin{array}{l}\text { Sca } \\
\text { Sca } \\
\text { Sca } \\
\text { Sca }\end{array}$ & $\begin{array}{l}Z=Z_{\odot} \\
Z=Z_{\odot} \\
Z=Z \odot \\
Z=Z \odot\end{array}$ \\
\hline \multicolumn{8}{|c|}{ Buzzoni (2000): } \\
\hline $\mathrm{E}$ & 15 Gyr & 1.48 & 0.94 & 3.27 & single burst & Sal & $\begin{array}{l}\text { two-zone model, } \\
L_{\text {bol }}(\text { halo:bulge })=(0.15: 0.85)\end{array}$ \\
\hline $\mathrm{Sb}$ & $15 \mathrm{Gyr}$ & 0.58 & 0.63 & 2.79 & $\propto t^{-0.5}($ disk $)$ & Sal & $\begin{array}{l}\text { three-zone model } \\
\left.L_{\text {bol }} \text { (halo:bulge:disk }\right)=(0.05: 0.30: 0.65)\end{array}$ \\
\hline $\mathrm{Sc}$ & 15 Gyr & 0.36 & 0.53 & 2.62 & $\propto t^{-0.1}($ disk $)$ & Sal & $\begin{array}{l}Z(\text { halo }+ \text { bulge })=Z_{\odot}, Z(\text { disk })=Z \odot / 3 \\
\text { three-zone model } \\
L_{\text {bol }}(\text { halo:bulge:disk })=(0.03: 0.15: 0.82)\end{array}$ \\
\hline $\operatorname{Im}$ & 15 Gyr & 0.16 & 0.43 & 2.39 & $\propto t^{+0.8}$ & Sal & $\begin{array}{l}\bar{Z}(\text { halo }+ \text { bulge })=Z_{\odot}, Z(\text { disk })=Z_{\odot} / 3 \\
Z=Z_{\odot} / 3\end{array}$ \\
\hline \multicolumn{8}{|c|}{ Fioc \& Rocca Volmerange (1997): } \\
\hline $\mathrm{E}$ & 15 Gyr & 1.40 & 0.91 & 3.19 & Schmidt law & $\mathrm{R} \& \mathrm{~B}$ & $\begin{array}{l}\text { Schmidt parameters }\left(n, \nu^{-1}\right)=(1,0.2 \mathrm{Gyr}) \\
Z=Z_{\odot}\end{array}$ \\
\hline $\mathrm{Sb}$ & 12 Gyr & 0.92 & 0.73 & 3.14 & Schmidt law & $\mathrm{R} \& \mathrm{~B}$ & $\begin{array}{l}\text { Schmidt parameters }\left(n, \nu^{-1}\right)=(1,2.8 \mathrm{Gyr}) \\
Z=Z_{\odot}\end{array}$ \\
\hline $\mathrm{Sc}$ & $12 \mathrm{Gyr}$ & 0.59 & 0.58 & 2.94 & Schmidt law & $\mathrm{R} \& \mathrm{~B}$ & $\begin{array}{l}\text { Schmidt parameters }\left(n, \nu^{-1}\right)=(1,10.0 \mathrm{Gyr}), \\
Z=Z_{\odot}\end{array}$ \\
\hline $\operatorname{Im}$ & 4 Gyr & 0.20 & 0.36 & 2.26 & Schmidt law & $\mathrm{R} \& \mathrm{~B}$ & $\begin{array}{l}\text { Schmidt parameters }\left(n, \nu^{-1}\right)=(1,20.0 \mathrm{Gyr}), \\
Z=Z_{\odot}\end{array}$ \\
\hline \multicolumn{8}{|c|}{ Coleman et al. (1980): } \\
\hline $\mathrm{E}$ & - & 1.49 & 0.93 & - & - & - & M 31 model \\
\hline $\mathrm{Sbc}$ & - & 0.59 & 0.58 & - & - & - & - \\
\hline Scd & - & 0.37 & 0.49 & - & - & - & - \\
\hline $\operatorname{Im}$ & - & -0.06 & 0.32 & - & - & - & - \\
\hline \multicolumn{8}{|c|}{ Leitherer et al. (1999): } \\
\hline Starburst \#1 & $800 \mathrm{Myr}$ & -0.27 & 0.13 & 1.62 & const. & Sal & $Z=Z_{\odot}$ \\
\hline Starburst \#2 & $500 \mathrm{Myr}$ & -0.37 & 0.09 & 1.62 & const. & Sal & $Z=Z \odot$ \\
\hline Starburst \#3 & $100 \mathrm{Myr}$ & -0.66 & 0.02 & 1.70 & const. & Sal & $Z=Z_{\odot}$ \\
\hline Starburst \#4 & $50 \mathrm{Myr}$ & -0.76 & 0.00 & 1.72 & const. & Sal & $Z=Z \odot$ \\
\hline
\end{tabular}

${ }^{(*)}$ Sal $=$ Salpeter (1955), Sca $=$ Scalo (1986), R\&B = Rana \& Basu (1992).

Sawicki et al. (1997), we extended the CWW empirical library by adding two starburst models from Leitherer et al. (1999) at $t=50$ and 500 Myr. This enlarged dataset will hereafter be referred to as the CWW-extended (CWWE) set.

\subsection{ISM and IGM corrections}

Fluxes provided by synthetic SED models have to be corrected for ISM and IGM absorption effects. Both are wavelength dependent, the latter is also redshift dependent, and they can dramatically change the galaxy spectrum in the UV restframe.

Interstellar dust attenuation depends on the color excess parameter $E(B-V)$, scaling with dust column density, as well as on the differential ISM opacity $k(\lambda)$, which is related to the physical properties of dust grains. The classical relationship for intrinsic and emerging flux is:

$F_{\text {obs }}(\lambda)=F_{0}(\lambda) 10^{-0.4 E(B-V) k(\lambda)}$

In our work we adopted the Calzetti (1999) dust attenuation law $k(\lambda)$ (see Fig. 2, panel $a$ ) including grain absorption and scattering. The $E(B-V)$ parameter can take the values $0.0,0.05,0.1,0.2,0.3$. By definition, the empirical CWW spectra already include internal reddening.

We describe the IGM absorption as a function of redshift following Madau (1995) (Fig. 2, panel $b$ ).

To give an illustrative example of the combined action of dust and IGM, in Fig. 3 the BUZ Sb template has been reddened by different amounts of the color excess $E(B-V)$ up to 0.3 magnitudes (upper panel). The effect of the IGM 


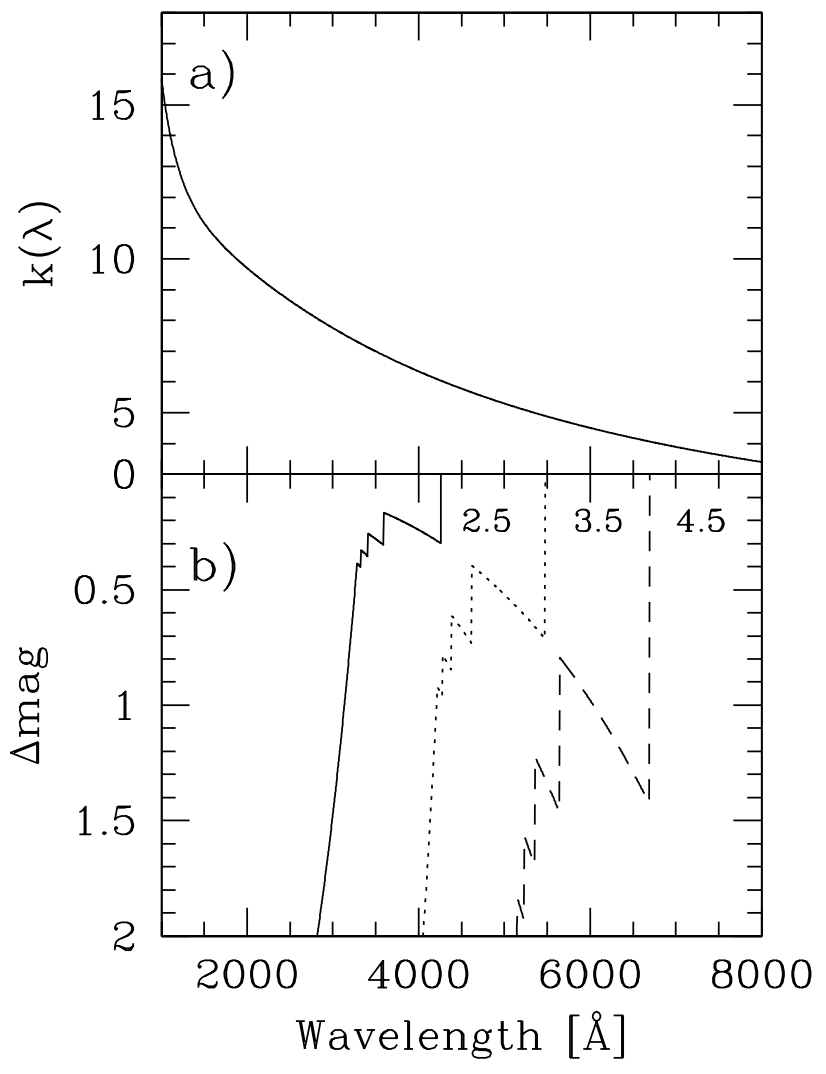

Fig. 2. Panel a) the adopted dust attenuation law after Calzetti (1999). Panel b) the IGM attenuation in a magnitude scale at redshift 2.5, 3.5 and 4.5, as labelled, according to Madau (1995)

on the same spectrum is displayed on the contrary in the lower panel for the two cases of redshift $z=1.5$ and $z=3$.

The galaxy spectral break induced by the cosmological Ly $-\alpha$ forest turns out to be the most effective feature to estimate redshifts beyond $z \sim 2$. The so-called "dropout" technique has been widely used to select high-redshift objects in current deep surveys (Steidel et al. 1995; Madau et al. 1996). Notice though that for faint objects the strong signature due to the IGM external feature can be confused with the $4000 \AA$ Balmer break (which, on the contrary is intrinsic to galaxy SED) resulting in a biased redshift estimate.

\section{Matching the FSLY catalog}

The HDFN photometric catalog produced by FSLY will be used as a target dataset for our analysis. It has been widely adopted in the recent literature (see, for instance, Arnouts et al. 1999; FSLY; Benítez 2000) and consists of 1067 objects observed with HST WFPC2 in the four photometric bands $U_{300}, B_{450}, V_{606}$, and $I_{814}$, and with the IRIM camera at KPNO in the infrared range (Johnson $J, H$, and $K$, Dickinson et al. 2000). The FSLY catalog contains fluxes, $f_{i}^{\text {obs }}$, and errors, $\sigma_{i}$, for each galaxy in all photometric bands $(i=1, \ldots 7)$. These quantities are those used in Eqs. (1)-(2).

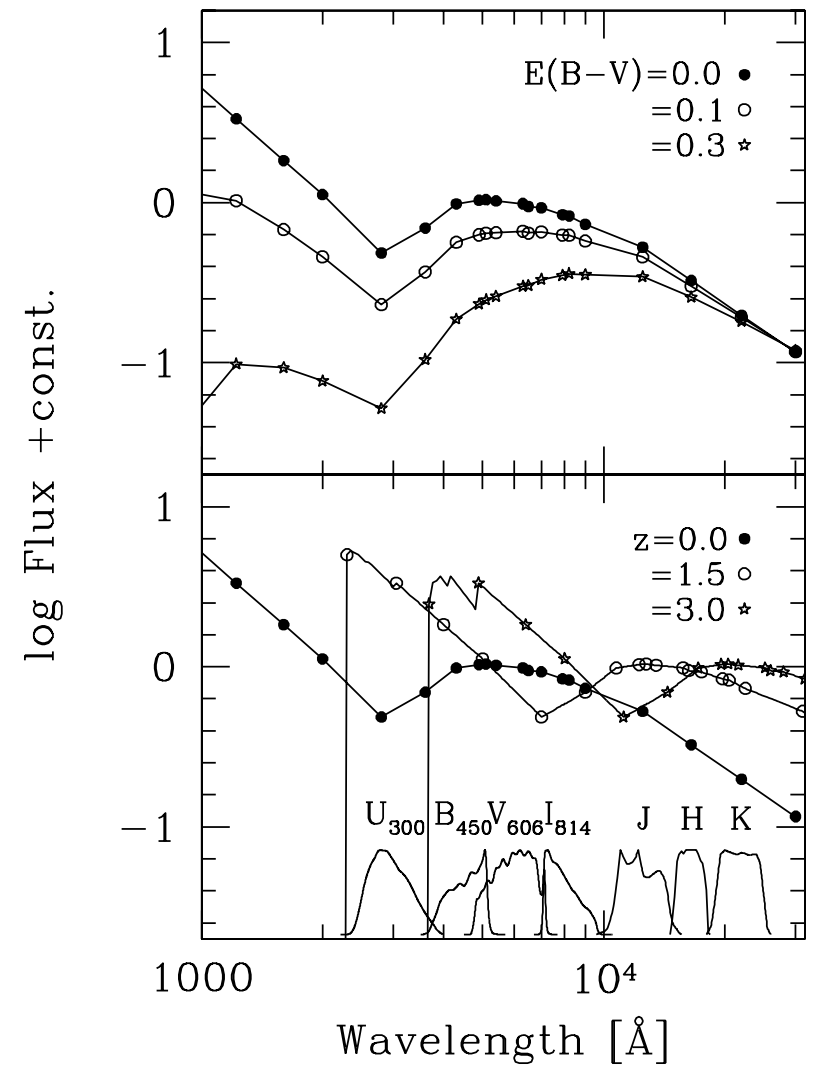

Fig. 3. The effect of internal reddening and IGM on the Sb reference template from Buzzoni (2000). Dust attenuation for $E(B-V)$ up to $0.3 \mathrm{mag}$, as labelled, is shown in the upper panel, while the expected break induced by the Ly- $\alpha$ forest at $z=1.5$ and $z=3$ is shown in the lower panel. For reference, the HST photometric system and the Johnson $J H K$ bands are displayed at the bottom

The availability of IR observations is of special relevance for our analysis because, even for galaxies at $z \sim 4$, it allows us to sample restframe emission at optical wavelengths, longward of the Balmer break. This greatly improves our chances to correctly interpret galaxy colors and obtain a fair redshift estimate for the most distant objects. As we can see in Fig. 1, within the photometric errors, both elliptical and blue star-forming galaxies at $z \sim 2$ show basically the same SED within the wavelength range covered by the HST photometric system. Only IR data can allow us to break color degeneracy.

For our analysis we selected from the original FSLY dataset only those objects with good photometry, that is with $S / N>5$ in at least two bands, in order to rely confidently on at least one color. Out of a total of 1067 galaxies, we were left with 1041 objects in our subsample. As a result of our selection we will be biased against the most distant objects beyond $z>5$. For these galaxies the Ly- $\alpha$ break enters the $V_{606}$ band, making them detectable only in the $I_{814}$ band (IR observations are not deep enough, in any case, to grant a good $S / N$ detection for these distant objects). 
Table 2. Statistical uncertainty in redshift estimate due to the photometric errors for each reference library (ten bootstrap simulations)

\begin{tabular}{lrcccc}
\hline & $\overline{\Delta z}$ & $\sigma_{z}$ & $\sigma_{z}$ & $|\Delta z|>0.5$ & $|\Delta z|>1.0$ \\
& & $z<1.5$ & $z>1.5$ & & \\
\hline BUZ & -0.01 & 0.16 & 0.24 & $11 \%$ & $7 \%$ \\
BC & -0.01 & 0.16 & 0.23 & $12 \%$ & $8 \%$ \\
FRV & -0.01 & 0.17 & 0.21 & $11 \%$ & $7 \%$ \\
CWWE & 0.00 & 0.15 & 0.26 & $12 \%$ & $8 \%$ \\
\hline
\end{tabular}

The $4000 \AA$ Balmer break being one of the most useful spectral feature for photometric redshift determination, we will single out a "low-redshift" subsample, including those galaxies for which the break is still within the HST photometric bands (this happens for $z<1.5$ ), and a "high-redshift" subsample $(z>1.5)$ for which the $4000 \AA$ break enters the IR photometric bands.

\subsection{Internal uncertainty of reference libraries}

It is of special relevance for our aim to preliminarily assess the effect of the observational uncertainties on the accuracy of redshift determination with the SED fitting method.

We used a simple bootstrap procedure to generate ten copies of the FSLY catalog, obtained by adding to each galaxy flux a correction randomly extracted from the Gaussian distribution of its photometric error.

For each of the reference template libraries we then compared, for each object, the redshift obtained from the measured photometry $\left(z_{\text {phot }}\right)$ and that from the bootstrapped galaxy photometry $\left(z_{\text {sim }}\right)$.

We will eventually have:

$\overline{\Delta z}=\sum_{N_{\mathrm{sim}}} \frac{\left(z_{\mathrm{phot}}-z_{\mathrm{sim}}\right)}{N_{\mathrm{sim}}}$

and

$\sigma_{z}^{2}=\sum_{N_{\mathrm{sim}}} \frac{\left[z_{\mathrm{phot}}-z_{\mathrm{sim}}\right]^{2}}{N_{\mathrm{sim}}}$.

where $N_{\text {sim }}=10 \times 1041$ is the number of simulated galaxies.

Moreover, we will check for the fraction of "discrepant" $\left(\left|z_{\text {phot }}-z_{\text {sim }}\right|>0.5\right)$ and "catastrophic" ( $\mid z_{\text {phot }}-$ $z_{\text {sim }} \mid>1.0$ ) outliers in the low- and high-redshift galaxy subsamples.

Table 2 gives a summary of the bootstrap results obtained for each theoretical synthesis code as well as for the CWWE empirical templates. Figure 4 shows in addition the distribution of the redshift residuals.

Since photometric errors increase at fainter magnitudes, redshift uncertainty is more pronounced for the most distant objects: $\sigma_{z} \sim 0.16$ at $z<1.5$, while $\sigma_{z} \sim 0.25$ for the $z>1.5$ subsample. The catastrophic outliers are always less than $10 \%$ of the global sample, and there are no systematic effects, as expected.

In addition to the photometric errors, also physical degeneracies in the $z$ vs. color space domain of the models could themselves result in a source of numerical instability for the redshift estimates. In this regard, different model sets could in principle provide also different "bias patterns" in the redshift distribution.

Quite interestingly, Table 2 shows that this is not the case for the four template sets accounted here. This might indicate that all reference libraries contain the same kind of degeneracy. In other words the galaxy colors are interpreted in the same way (from a statistical point of view) by the different template sets.

In Fig. 4 the distribution of redshift residuals for catastrofic outliers $(|\Delta z|>1.0)$ has a well defined pattern, and it is actually possible to identify two main sources for this pattern. The first is the color degeneracy between redshift $z \sim 0$ and $1.3<z<2.1$, that, given the photometric bands available in FSLY, shows up in all the templates. In the redshift interval $1.3<z<2.1$, the entire Lyman series has not yet entered the $U_{300}$ band (cf. Fig. 2), while the Balmer break is located between the $I_{814}$ and $J$ bands. The uncertainty in the photometric observations can easily cause a featureless objects to move within the ranges $z \sim 0$ and $1.3<z<2.1$. The second major source of the pattern visible in Fig. 4 are the biased $z$ estimates occuring when, due to large photometric errors, the Balmer break is mistaken for the Lyman decrement or vice versa. When the photometric errors in the IR bands are large enough to diluite/enhance a real/fictitious Balmer decrement, the objects affected are shuffled between the redshift ranges $0<z<1$ and $z>2.5$.

\subsection{Comparison among reference libraries}

We can now compare photometric redshifts obtained using different reference template libraries.

Given two independent redshift estimates, namely $z_{\text {phot, } 1}$ and $z_{\text {phot,2 }}$ from different libraries, we can again define a simple statistical moment of the residual distribution in the form:

$\overline{\Delta z}=\sum_{N_{\mathrm{g}}} \frac{\left(z_{\mathrm{phot}, 1}-z_{\mathrm{phot}, 2}\right)}{N_{\mathrm{g}}}$

while a variance can be computed as

$\sigma_{z}^{2}=\sum_{N_{\mathrm{g}}} \frac{\left[z_{\mathrm{phot}, 1}-z_{\mathrm{phot}, 2}\right]^{2}}{N_{\mathrm{g}}}$

where $N_{\mathrm{g}}=1041$ is the number of galaxies.

Also in this case, we should check for the fraction of discrepant $\left(\left|z_{\mathrm{phot}, 1}-z_{\mathrm{phot}, 2}\right|>0.5\right)$ and catastrophic $\left(\left|z_{\text {phot }, 1}-z_{\text {phot }, 2}\right|>1.0\right)$ outliers in the low- and highredshift galaxy subsamples.

In addition, we should also test whether $\overline{\Delta z}$ is consistent with zero or there is some systematic drift in data 

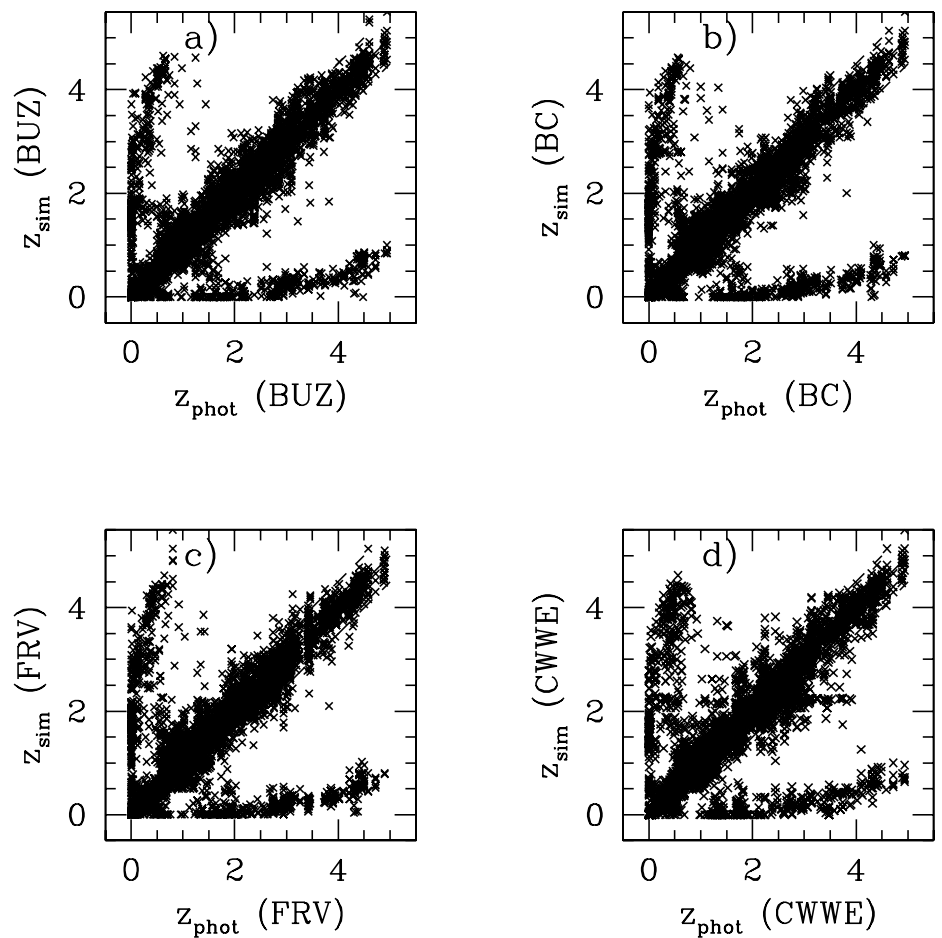

Fig. 4. Comparison of the statistical uncertainty in the redshift estimate due to photometric errors for the BC, BUZ and FRV reference libraries, as well as for the CWWE empirical templates. Residuals are from a bootstrap simulation of the FSLY galaxy catalog (1041 objects for each of ten simulated catalog samples)

Table 3. Comparison of redshift distributions from the different reference libraries

\begin{tabular}{|c|c|c|c|c|c|}
\hline & $\overline{\Delta z}$ & $\begin{array}{c}\sigma_{z} \\
z<1.5\end{array}$ & $\begin{array}{c}\sigma_{z} \\
z>1.5\end{array}$ & $|\Delta z|>0.5$ & $|\Delta z|>1.0$ \\
\hline BC vs. FRV & 0.02 & 0.09 & 0.12 & $3 \%$ & $3 \%$ \\
\hline BC vs. CWWE & 0.03 & 0.12 & 0.17 & $6 \%$ & $5 \%$ \\
\hline BUZ vs. BC & 0.00 & 0.12 & 0.13 & $4 \%$ & $3 \%$ \\
\hline BUZ vs. FRV & 0.02 & 0.13 & 0.15 & $5 \%$ & $4 \%$ \\
\hline BUZ vs. CWWE & 0.02 & 0.15 & 0.19 & $6 \%$ & $4 \%$ \\
\hline FRV vs. CWWE & 0.01 & 0.12 & 0.18 & $6 \%$ & $5 \%$ \\
\hline
\end{tabular}

residuals. Mean standard deviation should be explored against possible trends as a function of galaxy redshifts.

Figure 5 and Table 3 summarize the relevant results. The agreement between BC, BUZ and FRV (panels $a-c$ in Fig. 5) seems quite good, with less than $5 \%$ of catastrophic outliers, and $\sigma_{z}$ below 0.15 over the entire redshift range.

The comparison with the CWWE template set gives slightly poorer values for $\sigma_{z}$ (cf. panel $d$ in Fig. 5), especially for the most distant galaxies in the FSLY sample, but again a small fraction of discrepant redshift objects.

As we can appreciate in Table 3 , there are only marginal systematic drifts in the $\overline{\Delta z}$ residuals.

It should be noted that in the synthetic libraries the "primeval" evolutionary scenario is described with the same templates (the four starburst models from Leitherer et al. 1999). On the other hand, even by replacing, for example, the "primeval" BC library with the young galaxies provided by the $\mathrm{BC}$ code itself, results of the comparison with other models are left almost unchanged.

Comparing the results of Table 3 with those of Table 2, it is clear that the whole SED fitting procedure is largely insensitive to any specific choice of the model reference library. Rather than depending on the detailed galaxy $\mathrm{SED}$, the redshift is mainly constrained by the recognition of the Balmer and/or the Ly- $\alpha$ break in the galaxy flux distribution.

As a quite extreme but instructive example, in Fig. 6 we tried a fit of the galaxy subsample with fiducial redshift $z>2$ (259 objects, as estimated in every one of our reference libraries) using as a template set two Kurucz' (1992) model atmospheres for stars of $T=27000$ and $18000 \mathrm{~K}$. These temperatures are roughly consistent with a $B 0-B 2$ spectral type like for stars of $10-20 M_{\odot}$ that dominate starburst galaxy luminosity in the early 10-100 Myr of life (Leitherer et al. 1999). The redshift distribution relying on this "minimal" template set well agrees with that obtained using, for example, the FRV model library $\left(\sigma_{z}=0.18\right.$ and no catastrophic outliers). At high redshifts, therefore, the SED fitting method provides robust estimates of galaxy redshifts, even when using an extremely poor library of templates. It is clear, however, that such a simplified procedure cannot be of any utility if we are interested in a more accurate analysis of high-redshift data, such as to investigate age distribution or galaxy morphology at different cosmological times.

Another interesting check of internal consistency of the SED fitting method deals with the age-redshift 

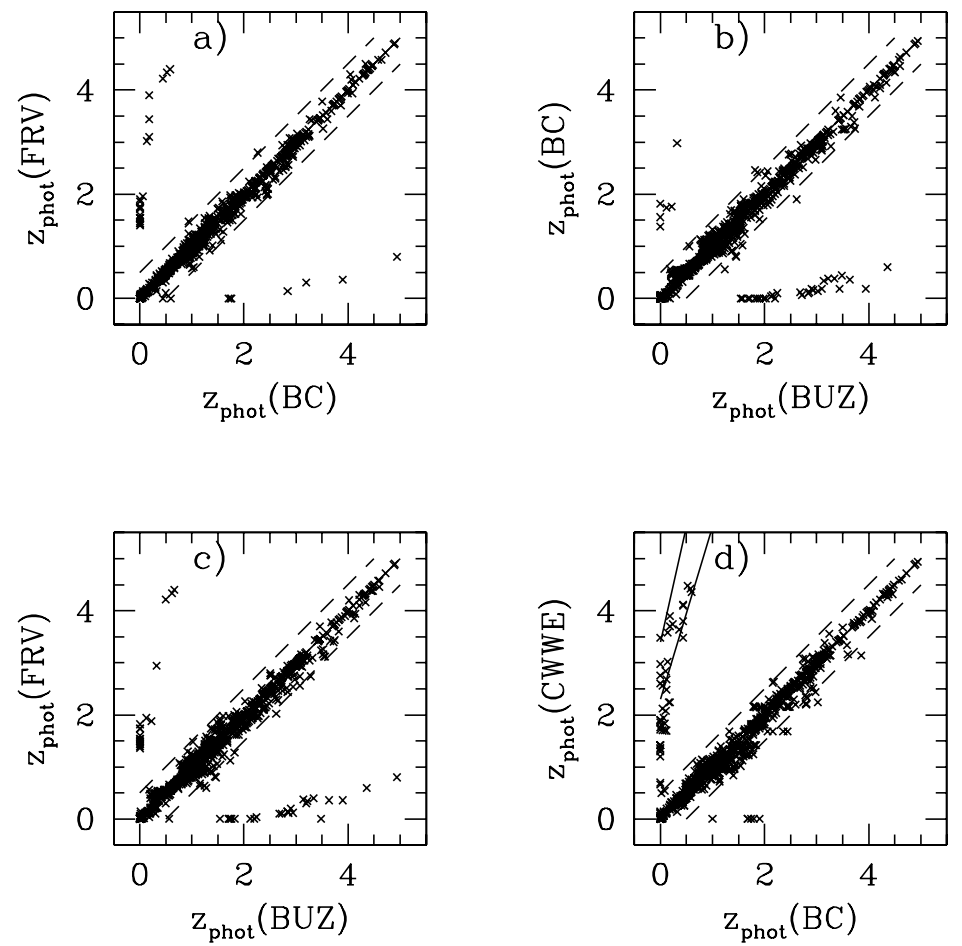

Fig. 5. Comparison of HDFN expected redshift distribution, for different reference libraries. The solid line is for $\Delta z=0.0$, while long-dashed strip is for $\Delta z= \pm 0.5$. The vertical strip (solid lines) in panel $d$ encloses the catastrophic outliers coming from the misinterpretation of the Balmer/Lyman break (see text for further details)

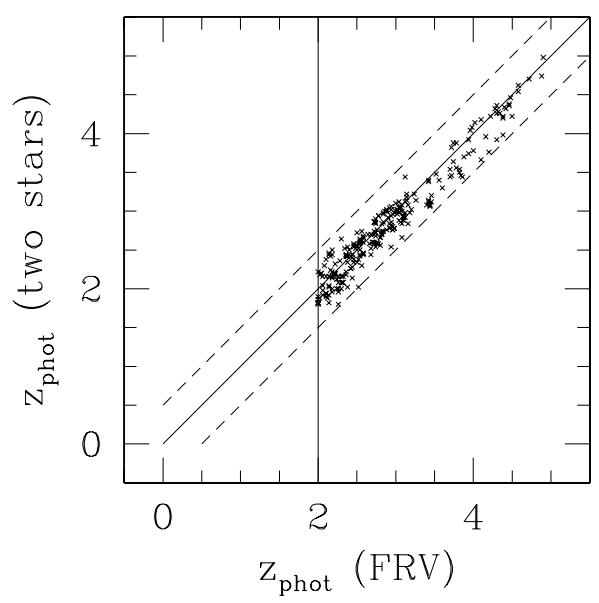

Fig. 6. SED fitting of the FSLY galaxy subsample beyond $z>$ 2. The data distribution by matching the FRV template set is compared with a "minimal" reference library consisting of two Kurucz' (1992) model atmospheres for stars of spectral type $B 0$ and $B 2$. The relative $\sigma_{z}$ results 0.18 with no apparent drift in the data distribution. The solid line is for $\Delta z=0.0$, while long-dashed strip is for $\Delta z= \pm 0.5$. See text for a full discussion

constraint. We discarded such constraint, by allowing the procedure to choose also galaxy models older than the Hubble time. Plotting the galaxy age information and the redshift information, both obtained from the photometric redshift algorithm, will allow to check for their reciprocal consistency.
For this exercise we grouped HDFN galaxies such as to guarantee at least 100 objects per redshift bin. Figure 7 reports our results according to the BC, BUZ and FRV codes.

Over the whole sample, the percentages of the fitting templates older than the actual Hubble time $\left(\left[H_{0}, q_{0}\right]=\right.$ $[50,0.0])$ with BC, BUZ and FRV codes are $3.9 \%, 8.5 \%$ and $5.7 \%$ respectively. For each theoretical code the empirical redshift-age relation is virtually compatible with any cosmological model with $H_{0}=50$ and $q_{0}<0.5$.

\subsection{Catastrophic outliers}

Although wide general agreement exists among template libraries in predicting galaxy redshift, it is worth investigating in some detail the few striking cases of catastrophic discrepancies (i.e. $|\Delta z|>1.0$ ).

There are basically two groups of such outliers, as in the distribution of redshift residuals due to photometric errors. The first group consists of objects with featureless spectrum, that is with no significant break signature (within the photometric errors). This is typically the case of the faintest blue objects in the HDFN sample. Due to color degeneracy between redshift $z \sim 0$ and $1.3<z<2.1$, these galaxies are shuffled within the two intervals by different libraries. The redshift interval $1.3<z<2.1$ is intrinsically critical for the SED fitting algorithm given the photometric bands available to us. As already noted above, while the entire Lyman series has not yet entered the $U_{300}$ band (cf. Fig. 2), the Balmer 


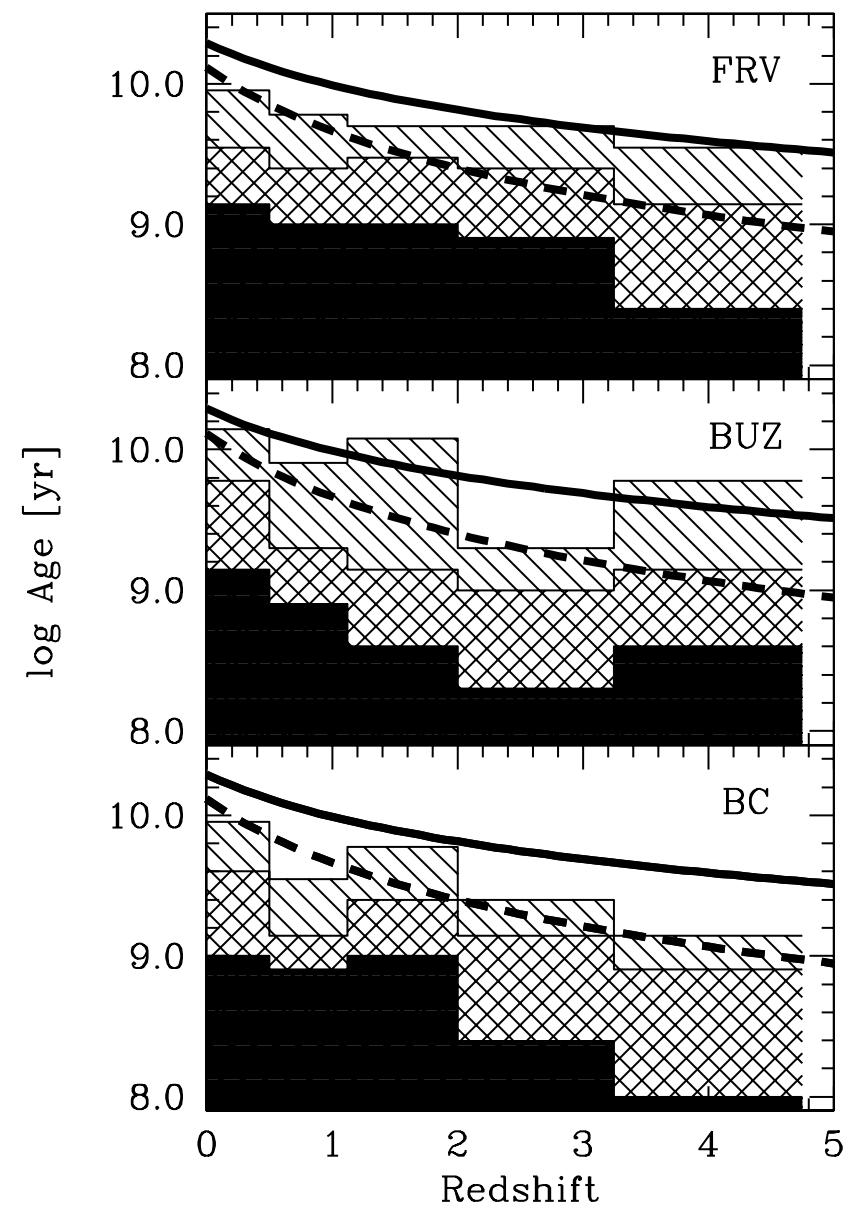

Fig. 7. The expected age-redshift relation from the BC, BUZ and FRV best templates by imposing no cosmological constraint. HDFN galaxies have been grouped in order to guarantee at least 100 objects per redshift bin. Solid, grid, and diagonal-shaded histograms are the 50\%, 75\%, and $90 \%$ envelopes of galaxy age distribution. In each plot, solid and dotted lines are the theoretical age- $z$ relation for $H_{0}=50$ and $q_{0}=0.0$ and 0.5 , respectively

break is located between the $I_{814}$ and $J$ bands. Therefore, redshift estimate entirely relies on the recognition of the smooth SED, and little changes in the reference templates can easily lead to diverging data interpretations.

The second class of catastrophic outliers comprises those objects for which the Balmer break is mistaken for the Lyman decrement or vice versa, according to the template library chosen, generating a series of biased $z$ estimates beyond $z>2.5$. This effect is especially evident when comparing CWWE vs. BC (cf. panel $d$ in Fig. 5). A too scanty sampling of galaxy morphology, like in the CWWE templates, is the cause of the relative exacerbation of the effect. For example galaxies with no deep Balmer break and relatively blue optical colors can be interpreted as Lyman break galaxies, in absence of alternative intermediate age elliptical templates.

More in general, a major source of catastrophic errors in the SED fitting procedure can result from the lack of starburst templates in the galaxy reference library.

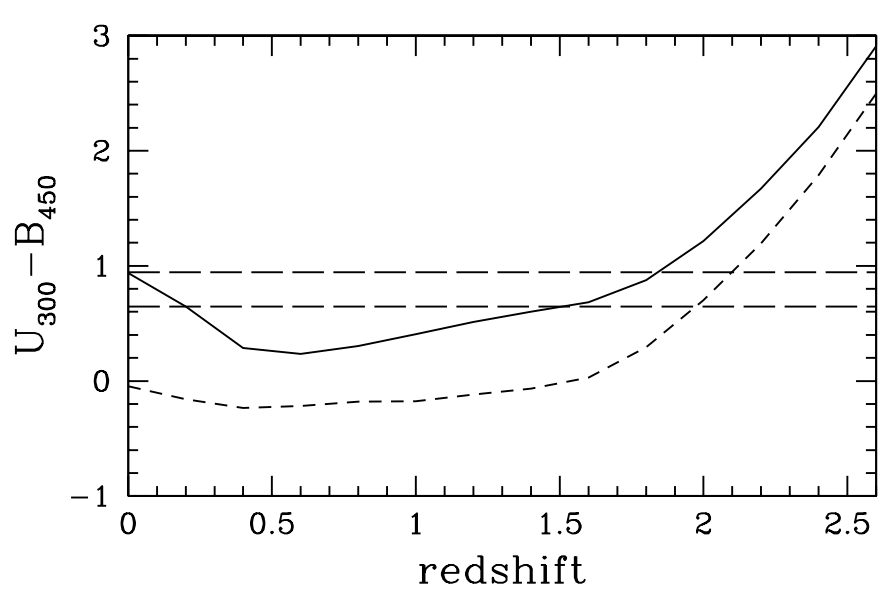

Fig. 8. The $U_{300}-B_{450}$ apparent color as a function of redshift for the CWW Im empirical template (solid line) and Leitherer et al. (1999) 50 Myr starburst model (dashed line). In absence of any starburst template, a fraction of star-forming galaxies at $z \sim 2$ (with $U_{300}-B_{450} \sim 0.8$ ) might be interpreted as local $(z \leq 0.2)$ irregulars

Standard Hubble types alone (even including Im irregulars) are not "blue" enough to reproduce the colors of the most active star-forming objects seen at high redshift. In Fig. 8 we show the $U_{300}-B_{450}$ apparent color of the CWWE Im template (solid line) and that of the Leitherer et al. (1999) 50 Myr starburst model (dotted line) as a function of redshift. Lacking starburst templates can introduce a systematic shift in the redshift estimate $(\Delta z \sim 0.3)$ for those galaxies with $\left(U_{300}-B_{450}\right) \sim 0.8$. Even worst, in some cases one might erroneously confuse UV-enhanced galaxies at $z \sim 2$ with local irregulars sampled in their optical SED. When comparing FSLY photometric redshifts with spectroscopic redshifts both this effects are evident.

\section{Starbursts and the role of ISM and IGM}

Dust reddening and IGM absorption have a large impact on the detection of starburst-galaxies at high redshifts, as these effects can effectively masque the original starburst spectrum.

In a simple experiment using the CWWE library and imposing $E(B-V)=0$ in our fit, about $20 \%$ of galaxies in the FSLY catalog can suitably be matched by (reddeningfree) starburst templates. When dust attenuation is accounted for, and $E(B-V)$ is left to vary as a free fitting parameter, this fraction raises to about $55 \%$ of the whole galaxy sample. A confident evaluation of the fraction of UV-enhanced galaxies at the different distances would have a pervasive impact in a number of cosmological problems, like for instance the study of the cosmic SFR (Madau et al. 1996; Steidel et al. 1999; Thompson et al. 2000).

To explore the selective influence of interstellar dust on the SED fitting procedure, we considered the FSLY subsample with fiducial $z>1.5$ (as estimated in every 


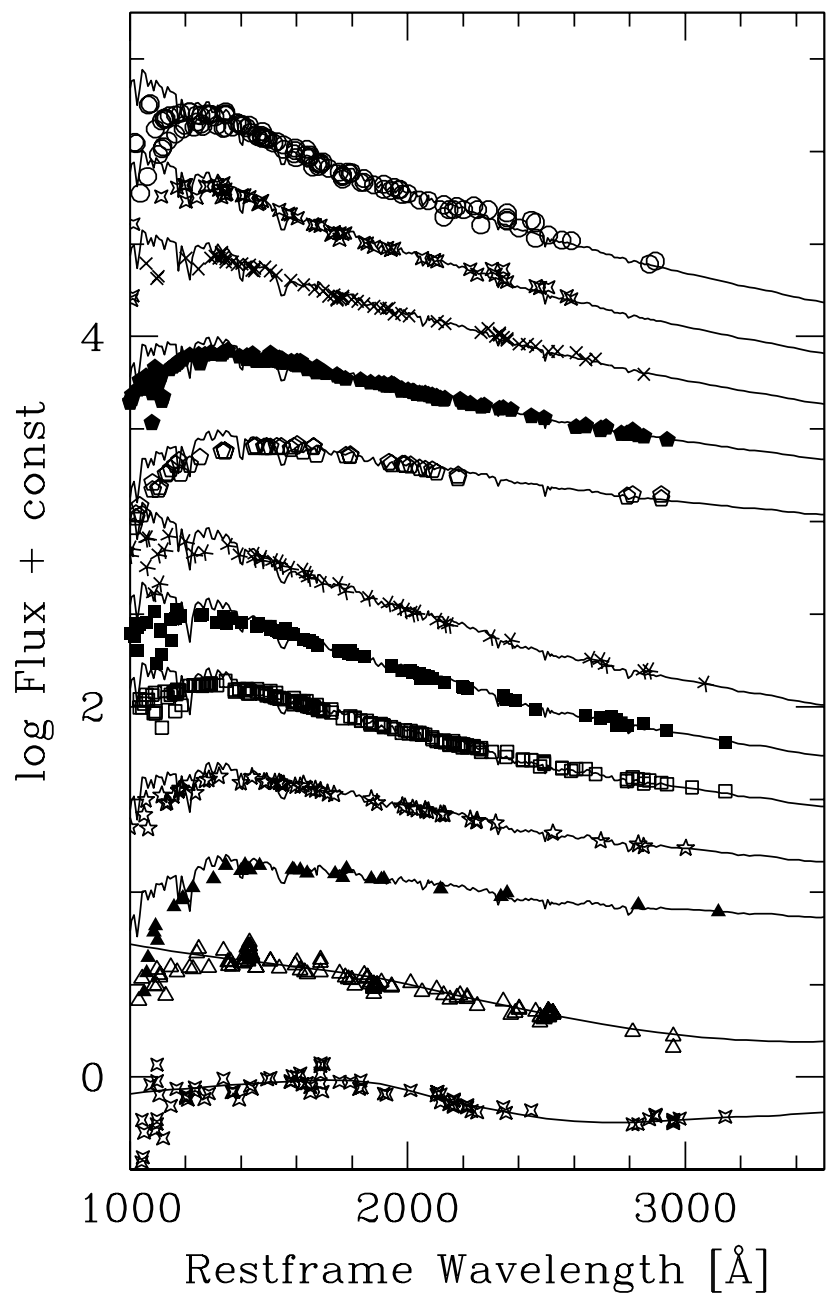

Fig. 9. Restframe composite SED from the HST data for the 341 HDFN galaxies with fiducial $z>1.5$ compared with the CWWE reference templates. From top to bottom solid lines display the starburst template with $t=500 \mathrm{Myr}$ and $E(B-$ $V)=0.0,0.05,0.1,0.2,0.3$, the $t=50$ Myr starburst model with $E(B-V)=0.0,0.05,0.1,0.2,0.3$, the $S c d$ and the Im CWW spectra

one of our reference libraries) and compared it with the CWWE empirical library (see Fig. 9).

Out of 348 selected galaxies, 341 can be fitted by starburst templates, $S c d$ spirals or later morphological types.

In a dust-free fitting scheme (i.e. by assuming $E(B-V)=0)$ we derive the following relative partition among fiducial morphological types:

\section{[others:Scd:Im:Starbursts] $=[7: 34: 160: 147]$}

with about $12 \%$ objects poorly fitted, that is at least $3-\sigma$ off in each band according to Eq. (1). Incidentally, if we exclude from the template library the starburst models we obtain:

[others:Scd:Im] $=[7: 43: 298]$

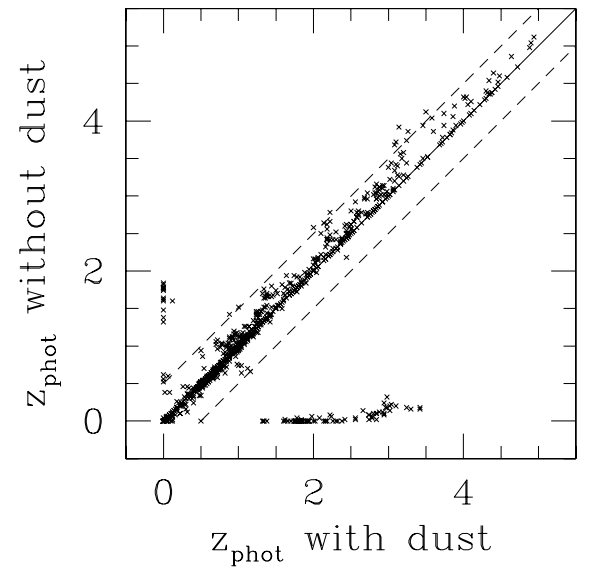

Fig. 10. The selective influence of galaxy internal reddening on the SED fitting performance. The HDFN redshift distribution from the $\mathrm{BC}$ template set is displayed with and without taking into account for ISM absorption. This is done by leaving $E(B-$ $V)=0$ or as a free fitting parameter in the range $0.0 \leq E(B-$ $V) \leq 0.3$. Long-dashed lines in the plot represent $\Delta z= \pm 0.5$

but at the cost of almost doubling the fraction of poorly fitted objects $(\sim 24 \%$ of the total sample).

If we leave $E(B-V)$ as a free fitting parameter (in the range $0.0 \leq E(B-V) \leq 0.3)$, the quality of the fit improves remarkably (only $5 \%$ of objects deviates by $3-\sigma$ or more), and an even larger number of starburst candidates is obtained:

[others:Scd:Im:Starbursts] $=[7: 25: 41: 275]$.

A dust-free fitting scheme also drastically increases the number of catastrophic outliers $(\sim 10 \%$ on the entire catalog, cf. Fig. 10), and at $z>1.5$ the redshift of reddened galaxies is overestimated by $\overline{\Delta z} \sim 0.2$ since IGM absorption, which is an increasing function of redshift, is forced to mimic color reddening.

In Fig. 9 the scatter of the HST data (blueshifted according the $z_{\text {phot }}$ value and normalized according the scaling factor $s$ of Eq. (2)) around the CWWE templates is shown directly. As we can see, at $\lambda>1216 \AA$ the best templates are an accurate description of high-redshift galaxy photometry. Notice however that at $\lambda<1216 \AA$ the observed fluxes deviate from the best templates: the experimental data do contain the IGM absorption, while the best templates do not.

The effect of IGM at high redshifts is explored in Fig. 11. Redshifts in the FSLY catalogue are determined using the BUZ reference library with and without IGM taken into account. A drift clearly appears beyond $z>1.5$ when the Ly $-\alpha$ break enters the $U_{300}$ band. The shift is generated by the wavelength difference between the $912 \AA$ Lyman break in the galaxy spectrum and the various steps of the $\mathrm{Ly}-\alpha$ forest. The fraction of outliers amounts to about $12 \%$ on the entire catalog and to $\sim 21 \%$ of the $z>1.5$ subsample. 


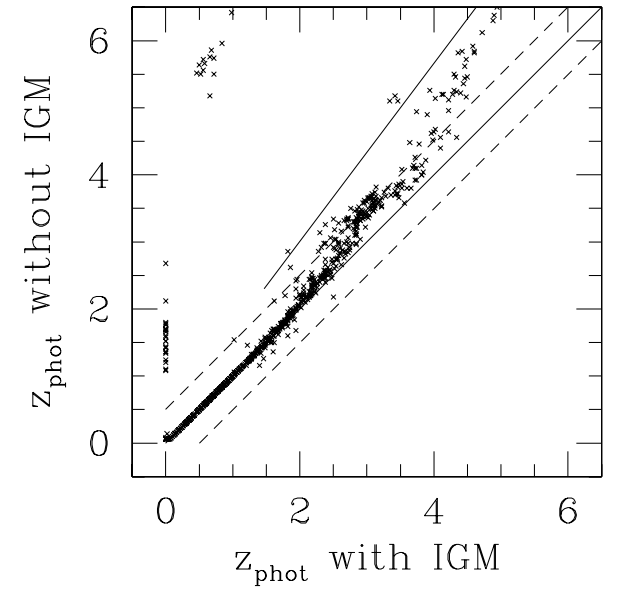

Fig. 11. Comparison of the HDFN redshift distribution obtained from the BUZ reference library with and without taking into account the IGM absorption. Long-dashed lines in the plot represent $\Delta z= \pm 0.5$. The solid line shows the expected upper limit in the redshift drift due to the the misinterpretation of the intrinsic Lyman break with the Ly- $\alpha$ forest effect

\section{Discussion and conclusions}

We investigated the stability of photometric redshift estimates in the SED fitting method with respect to the galaxy template set chosen. We used as a target dataset the FSLY catalog in the HDFN and as libraries of galaxy colors those produced by the theoretical codes of Bruzual \& Charlot (1993), Buzzoni (2000) and Fioc \& RoccaVolmerange (1997), as well as the empirical template set of Coleman et al. (1980). This way our model reference framework comprises (and enlarges) the sample of template libraries used by almost all the literature on the field.

The existence of non negligible photometric errors in the data is by itself an important source of uncertainty in the redshift estimates. Comparing the FSLY results with a number of bootstrap simulation of the same galaxy sample we estimate that the internal uncertainty in the SED fitting performance amounts to $\sigma_{z} \sim 0.16$ at $z<1.5$ and $\sigma_{z} \sim 0.25$ at $z>1.5$, with $\sim 8 \%$ of catastrophic outliers. This result is almost independent from the adopted model library and can be read as a lower limit to the intrinsic accuracy of the fitting method given the photometric errors of the FSLY catalogue.

The external redshift uncertainty, found by comparing results obtained using different theoretical models, is typically $\sigma_{z} \sim 0.11$ at $z<1.5$ and $\sigma_{z} \sim 0.13$ at $z>1.5$, with less than $4 \%$ of catastrophic outliers. Comparing theoretical models and the CWWE empirical set we obtained $\sigma_{z} \sim 0.13$ at $z<1.5$ and $\sigma_{z} \sim 0.18$ at $z>1.5$, with less than $5 \%$ of catastrophic outliers. Our results show that, given the photometric errors in the data, the SED fitting procedure is to a large extent almost insensitive to any specific choice of the model reference library.

The lack of starburst templates in the model library, on the other hand, introduces a systematic bias in the redshift estimate $(\Delta z \sim 0.3)$ and a sensible increase of catastrophic outliers as UV-enhanced galaxies at $z \sim 2$ can be confused with local irregulars sampled in their optical SED.

Not including in the fitting procedure ISM dust reddening or IGM opacity has a dramatic impact on the distribution of redshifts measured.

A dust-free fitting scheme drastically increases the number of catastrophic outliers $(\sim 10 \%$ on the entire cata$\log$ ) and at $z>1.5$ the redshift of dust reddened galaxies is overestimated by $\overline{\Delta z} \sim 0.2$. When dust attenuation is accounted for, the fraction of galaxies matched by starburst templates raises from $\sim 20 \%$ (by imposing $E(B-V)=0$ ) to $\sim 55 \%$ of the whole HDFN sample.

In an IGM-transparent fitting scheme a drift in the redshift estimates clearly appears beyond $z>1.5$ and the fraction of outliers amounts to $\sim 21 \%$ of the high-redshift subsample.

Our results suggest that, especially at high redshifts, the description of physical processes of photon absorption in the interstellar and in the intergalactic medium plays a key role in the SED fitting method for interpreting photometric data. Any specific choice of the templates, as long as these describe both normal and starburst galaxies, has on the contrary a minor effect on the performance of the method.

Acknowledgements. It is a pleasure to thank S. Charlot for useful suggestions, and the referee, R. I. Thompson, for his comments. M. Massarotti acknowledges financial support by Osservatorio Astronomico di Brera and Fondazione Cariplo.

\section{References}

Arnouts, S., Cristiani, S., Moscardini, L., et al. 1999, MNRAS, 310,540

Benítez, N. 2000, ApJ, 536, 571

Bruzual, G. A., Magris, G. C., \& Calvet, N. 1988, ApJ, 333, 673

Bruzual, G. A., \& Charlot, S. 1993, ApJ, 405, 538

Buzzoni, A. 1989, ApJS, 71, 817

Buzzoni, A. 1995, ApJS, 98, 69

Buzzoni, A. 1998, in IAU Symp. 183, Cosmological Parameters and the Evolution of the Universe, ed. K. Sato (Dordrecht, Kluwer), 134

Buzzoni, A. 2000, A\&A, submitted

Calzetti, D. 1999, Ap\&SS, 266, 243

Charlot, S., Worthey, G., \& Bressan, A. 1996, ApJ, 457, 625

Coleman, G. D., Wu, C. C., \& Weedman, D. W. 1980, ApJS, 43, 393

Connolly, A. J., Szalay, A. S., Dickinson, M., et al. 1997, ApJ, 486, 11L

Connolly, A. J., Szalay, A. S., \& Brunner, R. J. 1998, ApJ, 499, $125 \mathrm{~L}$

Cowie, L. L., Songaila, A., Hu, E. M., et al. 1996, ApJ, 112, 839

Dickinson, M., et al. 2000, in preparation

Ellis, R. S., Colless, M., Broadhurst, T., et al. 1996, MNRAS, 280,235

Fernández-Soto, A., Lanzetta, K. M., \& Yahil, A. 1999, ApJ, 513, 34 (FSLY) 
Fernández-Soto, A., Lanzetta, K. M., Chen, Hsiao-Wen, et al. 2000, ApJ, submitted [astro-ph/0007447]

Fioc, M., \& Rocca-Volmerange, B. 1997, A\&A, 326, 950

Franceschini, A., Silva, L., Fasano, G., et al. 1998, ApJ, 506, 600

Giallongo, E., D'Odorico, S., Fontana, A., et al. 1998, AJ, 115, 2169

Gordon, K. D., Calzetti, D., \& Witt, A. N. 1997, ApJ, 487, 625

Kuchinski, L. E., Terndrup, D. M., Gordon, K. D., et al. 1998, AJ, 115, 1438

Kurucz, R. 1992, in Proc. of the IAU Symp No. 149, The Stellar Populations of Galaxies, ed. A. Renzini, \& B. Barbuy (Kluwer, Dordrecht), 225

Leitherer, C., Schaerer, D., Goldader, J. D., et al. 1999, ApJS, 123,3

Lilly, S. J., Tresse, L., Hammer, F., et al. 1995, ApJ, 455, 108

Madau, P. 1995, ApJ, 441, 18

Madau, P., Ferguson, H. C., Dickinson, M., et al. 1996, MNRAS, 283, 1388

Miralles, J. M., \& Pelló, R. 1998, ApJ submitted [astro-ph/9801062]
Pascarelle, S. M., Lanzetta, K. M., \& Fernández-Soto, A. 1998, ApJ, 508, 1L

Rana, N. C., \& Basu, S. 1992, A\&A, 265, 499

Renzini, A., \& Buzzoni, A. 1986, in Spectral Evolution of Galaxies, ed. C. Chiosi, \& A. Renzini (Reidel, Dordrecht), 195

Salpeter, E. E. 1955, ApJ, 121, 161

Sawicki, M. J., Lin, H., \& Yee, H. K. C. 1997, AJ, 113, 1

Scalo, J. M. 1986, Fund. Cos. Phys., 11, 1

Steidel, C. C., Pettini, M., \& Hamilton, D. 1995, AJ, 110, 2519

Steidel, C. C., Adelberger, K. L., Giavalisco, M., et al. 1999, ApJ, 519, 1

Thompson, R. I., Weymann, R. J., \& Storrie-Lombardi, L. J. 2000, ApJ, in press [astro-ph/0008276]

Wang, Y., Bahcall, N., \& Turner, E. L. 1999, ed. R. Weymann, L. Storrie-Lombardi, M. Sawicki, \& R. Brunner, ASP Conf. Ser., 191, 25

Williams, R. E., Blacker, B., Dickinson, M., et al. 1996, AJ, 112,1335

Yee, H. K. C. 1998 [astro-ph/9809347] 